\title{
Finite Time Stabilization of the Four Tanks System: Extensions to the Uncertain Systems
}

\author{
Chakib Ben Njima, ${ }^{1}$ Walid Ben Mabrouk, ${ }^{1}$ Hassani Messaoud, ${ }^{1}$ and Germain Garcia ${ }^{2,3}$ \\ ${ }^{1}$ Ecole Nationale d'Ingénieurs de Monastir, Université de Monsatir, Rue Ibn El Jazzar, 5019 Monastir, Tunisia \\ ${ }^{2}$ CNRS, LAAS, 7 Avenue du Colonel Roche, 31077 Toulouse, France \\ ${ }^{3}$ Université de Toulouse, UPS, INSA, INP, ISAE, LAAS, 7 Avenue du Colonel Roche, F-31077 Toulouse, France
}

Correspondence should be addressed to Chakib Ben Njima; chakib.bennjima@enim.rnu.tn

Received 26 November 2013; Accepted 13 January 2014; Published 4 March 2014

Academic Editor: Hui Zhang

Copyright (C) 2014 Chakib Ben Njima et al. This is an open access article distributed under the Creative Commons Attribution License, which permits unrestricted use, distribution, and reproduction in any medium, provided the original work is properly cited.

\begin{abstract}
We consider the finite time stability and stabilization of linear systems described in continuous time. First, we provide a condition for the stability over time using the state transition matrix standard. Then we give conditions to design a state feedback control that stabilizes the system over time. In some cases where there is uncertainty in the system model, the previous conditions are extended to a certain class of uncertain systems. The considered uncertainties are the polytopic and norm bounded ones. To reveal the proposed approach, an application to the four tanks system was made.
\end{abstract}

\section{Introduction}

Several studies of the stability for linear uncertain systems called robust stability were carried out during the last two decades $[1,2]$. Most of the results in this field relating to the stability and performance criteria are defined on infinite time interval. However, in many practical applications, the main objective is the system's behavior on a finite time interval. Thus, it seems reasonable to define a stable system if its state is in the prescribed limits into a fixed time interval when starting from certain conditions, but in the opposite case we are talking about an instable system.

This kind of stability is called finite time stability or short time stability $[3,4]$, and there are many practical problems where it has an important role such as the control of a spatial trajectory of a spacecraft starting from an initial point to a finale one in a prescribed time interval. Finite time stabilization is still one of the major problems in control theory. Generally, the stabilization problems are treated under an asymptotic aspect, without considering the fact that the dynamic behavior of a system must match the human operations scale.

Even if we have the possibility to study the exponential convergence, it still presents a hard problem to solve when we are treating nonlinear systems. In practice, it is important to specify the performance of convergence, which means to make sure that the system joins a short and precise time reference or desired trajectory.

In the literature there are many works related to this topic $[5,6]$ known as finite time stability, but this concept differs from what is studied in this paper. Indeed, with this concept if a system is finite time stable, it assumes that it is already asymptotically stable but this is not the case in the notion that we study. This concept is highly demanding and it is very difficult to provide some conditions that help to solve the problem. But, it is possible to spread out this notion and to guarantee the finite time stability by ignoring the asymptotic aspect. In this case, we must ensure that the state is still bounded for a given time interval. The application's fields of the finite time stabilization methods are numerous; we can mention robotic control, chemical processes [4], the control of missiles [4], and so forth.

In this context, for some applications, it is better to ensure that the state is bounded during a time interval that matches to the needed time to execute a practical task instead of guaranteeing that the system is asymptotically stable, for example, the movement of manipulator from a point $\mathrm{A}$ to a point $\mathrm{B}$ through a nominal path or trajectory or kipping 
the pressure, the temperature, or another physical quantity during very precise period in a chemical process. In these practical situations, a process should be stable if its state evolves while being bounded by required limits; otherwise it is considered unstable. All references mentioned above are limited to the Finite Time Stability study and no results on the synthesis are known. Recently, [7-10] treated the case of linear systems where they solved the problem of the synthesis.

The main objective of the work reported in this paper is to provide an ordered framework to study the finite times stability and to synthesize the control laws that stabilize in finite time the uncertain linear systems. In this regard, the main contribution focuses on the analysis and synthesis of the finite time stability of the uncertain linear systems by solving Linear Matrix Inequalities (LMIs), where we propose a new method for a control law synthesizing by state feedback which is based on solving LMIs and stabilizes, finite time, the linear systems. An extension of this result to uncertain systems has been developed. First, we give the definitions corresponding to the finite time stability in terms of sets and terms of standards; then we will mention a new approach to the control laws by state feedback that stabilizes in finite time the linear continuous time systems solving some LMIs; this will be extended to cover uncertain systems where we will look for polytopic and norm bounded uncertainties. The method will be validated on a level control system with four tanks.

\section{Finite Time Stability}

Let us consider the system described by

$$
\dot{x}=A x,
$$

where $x$ is the state vector and $A \in \mathbb{R}^{n \times n}$ is the dynamic matrix.

System (1) has a unique solution of the following form:

$$
x(t)=e^{A\left(t-t_{0}\right)} x\left(t_{0}\right) .
$$

The finite time stability consists of maintaining the system state within prescribed bounds during a given time interval.

The following definition can be introduced $[11,12]$.

Definition 1. System (1) is finite time stable (FTS) with respect to $\left(S_{I}, S_{A},\left[t_{0}, t_{0}+T\right]\right)$ (Figure 1$)$ if

$$
x\left(t_{0}\right) \in S_{I} \Longrightarrow x(t) \in S_{A} \quad \forall t \in\left[t_{0}, t_{0}+T\right],
$$

where $S_{I}$ is the set of initial states and $S_{A}$ is the set of admissible ones that can be ellipsoids given by

$$
\begin{gathered}
S_{I}=\left\{x \in \mathbb{R}^{n}:|x| \leq c_{1}, c_{1}>0\right\}, \\
S_{A}=\left\{x \in \mathbb{R}^{n}:|x| \leq c_{2}, c_{2}>c_{1}>0\right\},
\end{gathered}
$$

where $c_{1}$ and $c_{2}$ define the bounds of state, and the relation (3) can be written as

$$
\left|x\left(t_{0}\right)\right| \leq c_{1} \Longrightarrow|x(t)| \leq c_{2} \quad \forall t \in\left[t_{0}, t_{0}+T\right] .
$$

In the following, we propose a theorem that provides the necessary and sufficient condition of FTS.

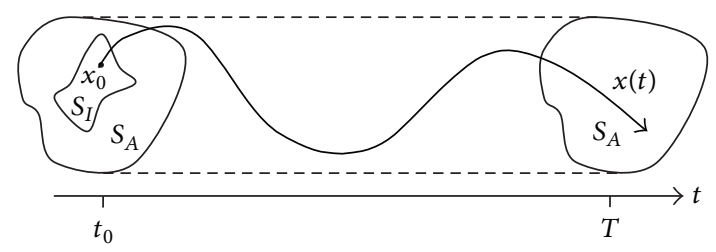

FIgURE 1: FTS with respect to $\left(S_{I}, S_{A},\left[t_{0}, t_{0}+T\right]\right)$.

Theorem 2. System (1) is finite time stable with respect to $\left(c_{1}, c_{2}, T\right)$ if and only if for all $t \in\left[t_{0}, t_{0}+T\right]$,

$$
\left\|e^{A\left(t-t_{0}\right)}\right\| \leq \frac{c_{2}}{c_{1}}
$$

which is also equivalent to

$$
\left\|e^{A^{T}\left(t-t_{0}\right)}\right\| \leq \frac{c_{2}}{c_{1}} .
$$

Proof. The sufficiency follows from the fact that $|B y| \leq$ $\|B\||y|$, where $B$ is a matrix and $y$ is a vector. Therefore, from (2),

$$
|x(t)|=\left\|e^{A\left(t-t_{0}\right)}\right\|\left|x\left(t_{0}\right)\right| .
$$

And from the first inequality (5), we have

$$
|x(t)|<\left\|e^{A\left(t-t_{0}\right)}\right\| c_{1} .
$$

If condition (6) of Theorem 2 is satisfied, then $|x(t)| \leq c_{2}$, which satisfies the second inequality of relation (5).

The necessity can be proved by contradiction as in [10].

Corollary 3. System (1) is finite time stable with respect to $\left(c_{1}, c_{2}, T\right)$ if and only if

$$
\lambda_{\text {max }}(P(t)) \leq \frac{c_{2}^{2}}{c_{1}^{2}} \quad \forall t \in\left[t_{0}, t_{0}+T\right],
$$

where $P(t)$ is a solution of the differential matrix equation

$$
\frac{d P}{d t}=A^{T} P+P A, \quad P\left(t_{0}\right)=I
$$

and $\lambda_{\max }(P(t))$ is the maximal eigenvalue of $P(t)$.

Proof. Define $P(t)=e^{A^{T}\left(t-t_{0}\right)} e^{A\left(t-t_{0}\right)}$.

We know that

$$
\|A\|=\sqrt{\lambda_{\max }\left(A^{T} A\right)} .
$$

Therefore,

$$
\lambda_{\max }(P)=\left\|e^{A\left(t-t_{0}\right)}\right\|^{2}
$$

If

$$
\lambda_{\max }(P)<\frac{c_{2}^{2}}{c_{1}^{2}} \Longrightarrow\left\|e^{A\left(t-t_{0}\right)}\right\|<\frac{c_{2}}{c_{1}},
$$

which concludes, from Theorem 2, the finite time stability. 
Even if they give necessary and sufficient conditions, the previous results are not easy to verify from a computational point of view, particularly the result stated in Theorem 2. It is possible to obtain more tractable conditions which are only sufficient.

Theorem 4. If there exist a positive definite symmetric matrix $P$ and a positive scalar $\beta$ such that

$$
0 \leq \beta T \leq \ln \left[\frac{c_{2}}{c_{1}}\right]
$$

and satisfying the following matrix inequalities:

$$
\begin{gathered}
A P+P A^{T}-\beta I \leq 0, \\
I \leq P \leq \frac{c_{2}^{2}}{c_{1}^{2}} e^{-\beta T} I,
\end{gathered}
$$

then system (1) is finite time stable with respect to $\left(c_{1}, c_{2}, T\right)$.

Proof. Suppose that conditions of theorem are satisfied. Differentiating $e^{A\left(t-t_{0}\right)} P e^{A^{T}\left(t-t_{0}\right)}$ with respect to time leads to

$$
\frac{d\left(e^{A\left(t-t_{0}\right)} P e^{A^{T}\left(t-t_{0}\right)}\right)}{d t}=e^{A\left(t-t_{0}\right)}\left(A P+P A^{T}\right) e^{A^{T}\left(t-t_{0}\right)} .
$$

From condition (16) we can write

$$
\frac{d\left(e^{A\left(t-t_{0}\right)} P e^{A^{T}\left(t-t_{0}\right)}\right)}{d t} \leq \beta e^{A\left(t-t_{0}\right)} e^{A^{T}\left(t-t_{0}\right)}
$$

and since $\lambda_{\min }(P) I \leq P$ where $\lambda_{\text {min }}(P)$ is the minimal eigenvalue of $P$,

$$
\frac{d\left(e^{A\left(t-t_{0}\right)} P e^{A^{T}\left(t-t_{0}\right)}\right)}{d t} \leq \frac{\beta e^{A\left(t-t_{0}\right)} P e^{A^{T}\left(t-t_{0}\right)}}{\lambda_{\min }(P)} .
$$

By Gronwall's Lemma [13] we obtain

$$
e^{A\left(t-t_{0}\right)} P e^{A^{T}\left(t-t_{0}\right)} \leq e^{\lambda_{\min }^{\beta}(P)^{t-t_{0}}} I, \quad t \geq t_{0},
$$

and from condition (17), we have $\lambda_{\min }(P) \geq 1$ and $\lambda_{\max }(P) \geq$ 1 .

Therefore,

$$
e^{A\left(t-t_{0}\right)} P e^{A^{T}\left(t-t_{0}\right)} \leq e^{\beta\left(t-t_{0}\right)} I, \quad t \geq t_{0} .
$$

We have also

$$
\lambda_{\min }(P) e^{A\left(t-t_{0}\right)} e^{A^{T}\left(t-t_{0}\right)} \leq e^{A\left(t-t_{0}\right)} P e^{A^{T}\left(t-t_{0}\right)},
$$

and then

$$
\begin{aligned}
& \frac{\lambda_{\min }(P)}{\lambda_{\max }(P)} e^{A\left(t-t_{0}\right)} e^{A^{T}\left(t-t_{0}\right)} \leq e^{A\left(t-t_{0}\right)} P e^{A^{T}\left(t-t_{0}\right)} \\
& \quad \leq e^{\beta\left(t-t_{0}\right)} I, \quad t \geq t_{0} .
\end{aligned}
$$

Since

$$
\lambda_{\max }\left(e^{A^{T}} e^{A}\right)=\left\|e^{A^{T}}\right\|^{2},
$$

we have

$$
e^{A\left(t-t_{0}\right)} e^{A^{T}\left(t-t_{0}\right)}<\frac{\lambda_{\max }(P)}{\lambda_{\min }(P)} e^{\beta\left(t-t_{0}\right)} I .
$$

Consequently,

$$
\lambda_{\max }\left(e^{A\left(t-t_{0}\right)} e^{A^{T}\left(t-t_{0}\right)}\right)<\frac{\lambda_{\max }(P)}{\lambda_{\min }(P)} e^{\beta\left(t-t_{0}\right)},
$$

where $\lambda_{\max }\left(e^{A\left(t-t_{0}\right)} e^{A^{T}\left(t-t_{0}\right)}\right)$ is the maximal eigenvalue of $e^{A\left(t-t_{0}\right)} e^{A^{T}\left(t-t_{0}\right)}$.

We deduce that

$$
\begin{gathered}
\left\|e^{A^{T}\left(t-t_{0}\right)}\right\|^{2} \leq \frac{\lambda_{\text {max }}(P)}{\lambda_{\text {min }}(P)} e^{\beta\left(t-t_{0}\right)}, \quad t>t_{0}, \\
\left\|e^{A^{T}\left(t-t_{0}\right)}\right\|^{2} \leq \frac{\lambda_{\text {max }}(P)}{\lambda_{\text {min }}(P)} e^{\beta T} \leq \frac{c_{2}^{2}}{c_{1}^{2}} \quad \forall t \in\left[t_{0}, t_{0}+T\right] \text { by }(6),
\end{gathered}
$$

which concludes the proof.

From the previous theorem, we can deduce the following interesting result.

Corollary 5. The conditions of Theorem 4 are satisfied if there exists a positive scalar $\gamma$ satisfying

$$
\begin{aligned}
& A+A^{T}-\gamma I \leq 0, \\
& \gamma T \leq 2 \ln \left[\frac{c_{2}}{c_{1}}\right] .
\end{aligned}
$$

Then system (1) is finite time stable with respect to $\left(c_{1}, c_{2}, T\right)$. In addition, for all values of $c_{1}, c_{2}$, and $T$ satisfying

$$
2 \ln \left[\frac{c_{2}}{c_{1}}\right] \geq T \lambda_{\max }\left(A+A^{T}\right),
$$

system (1) is finite time stable with respect to $\left(c_{1}, c_{2}, T\right)$.

Proof. Suppose that conditions of Corollary 5 are satisfied. Then $P=I$ and $\beta=\gamma$ satisfy conditions of Theorem 4 . In addition if the first inequality is satisfied, we have

$$
\gamma \geq \lambda_{\max }\left(A+A^{T}\right)
$$

and the last part of the corollary follows.

We can state the following result for the case of asymptotically stable systems.

Corollary 6. If there exists a positive definite symmetric matrix $P$ satisfying the Linear Matrix Inequalities (LMIs)

$$
A P+P A^{T}<0,
$$

$$
I \leq P \leq \frac{c_{2}^{2}}{c_{1}^{2}} I
$$

then system (1) is asymptotically and finite time stable with respect to $\left(c_{1}, c_{2}, T\right)$. 
Proof. Suppose that conditions of the corollary are satisfied. Following the proof of Theorem 2, we obtain

$$
\left\|e^{A^{T}\left(t-t_{0}\right)}\right\|^{2} \leq \frac{\lambda_{\max }(P)}{\lambda_{\min }(P)} e^{\beta\left(t-t_{0}\right)}, \quad t>t_{0} .
$$

Considering $\beta=0$, we obtain

$$
\left\|e^{A^{T}\left(t-t_{0}\right)}\right\|^{2} \leq \frac{\lambda_{\text {max }}(P)}{\lambda_{\min }(P)} \leq \frac{c_{2}^{2}}{c_{1}^{2}} \quad \forall t \in\left[t_{0}, t_{0}+T\right]
$$

which concludes the proof.

\section{Finite Time Stabilization}

We consider in this section the following system:

$$
\dot{x}=A x+B u, \quad x\left(t_{0}\right)=x_{0},
$$

where $A \in \mathbb{R}^{n \times n}$ and $B \in \mathbb{R}^{n \times m}$. The problem addressed in this paragraph can be stated as follows.

Problem 7. Find a state feedback control $u=K x$ with $K \in$ $\mathbb{R}^{m \times n}$ such that system (35) is finite time stable with respect to $\left(c_{1}, c_{2}, T\right)$.

From Theorem 2, it is possible to deduce the following result.

Theorem 8. If there exist a positive definite symmetric matrix $S$, a matrix $R$ of appropriate dimension, and a positive scalar $\beta$ such that

$$
0 \leq \beta T \leq \ln \left[\frac{c_{2}}{c_{1}}\right]
$$

and satisfying the matrix inequalities

$$
\begin{gathered}
A S+S A^{T}+B R+R^{T} B^{T}-\beta I \leq 0, \\
I \leq S \leq \frac{c_{2}^{2}}{c_{1}^{2}} e^{-\beta T} I,
\end{gathered}
$$

then the control law $u=R S^{-1} x$ solves Problem 7 .

Proof. The proof follows from the fact that inequality (37) can be written as

$$
\left(A+B R S^{-1}\right) S+S\left(A+B R S^{-1}\right)^{T}-\beta I \leq 0 .
$$

It is also possible to obtain directly a control law from the Corollary 9.

Corollary 9. If there exist a positive scalar $\gamma$ and a matrix $K$ of appropriate dimensions satisfying

$$
\begin{gathered}
A+B K+A^{T}+K^{T} B^{T}-\gamma I \leq 0, \\
\gamma T \leq 2 \ln \left[\frac{c_{2}}{c_{1}}\right],
\end{gathered}
$$

then the control law $u=K x$ solves Problem 7 with $K=R S^{-1}$.

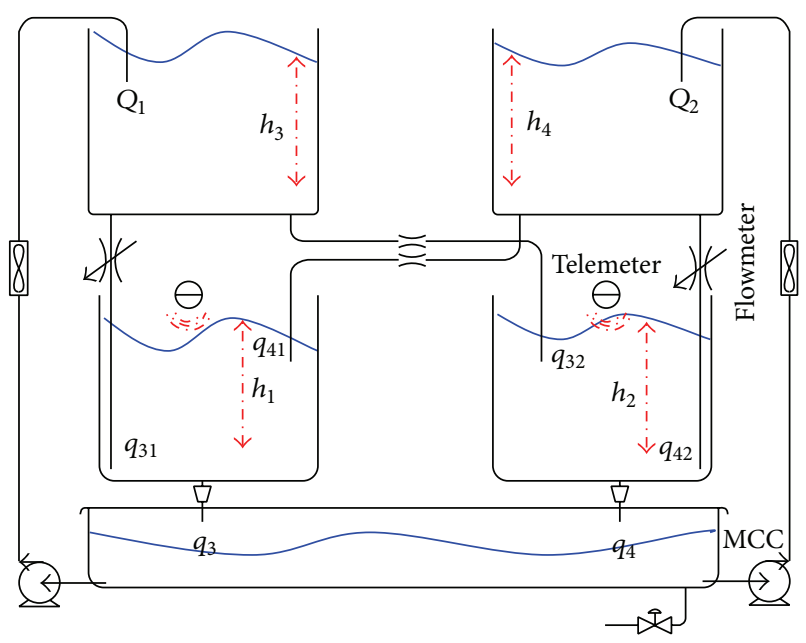

Figure 2: Four tanks system synoptic schematic.

\section{Application on Four Tanks System}

In this application, we test the finite time stabilization using the LMI method on the four tanks system. Since the considered system is nonlinear, it is linearised around an operating point and the state should be bounded around this point.

(A) System Modeling. For simplicity, we assimilate the four tanks system to that given by the block diagram (Figure 2).

The model of the process is achieved by generalization of one tank process model (Figure 3 ).

The process model is given by

$$
\frac{d h}{d t}=\frac{1}{S} q_{e}-\frac{s}{S} \sqrt{2 g h},
$$

where $q_{e}$ is the inflow, $h$ is the water level, $s$ is the section of leak, and $S$ is the section of tank. And the four tanks process model can be given as

$$
\begin{aligned}
& S \frac{d h_{1}}{d t}=q_{31}+q_{41}-q_{3}, \\
& S \frac{d h_{2}}{d t}=q_{32}+q_{42}-q_{4}, \\
& S \frac{d h 3}{d t}=Q_{1}-q_{31}-q_{32}, \\
& S \frac{d h_{4}}{d t}=Q_{2}-q_{41}-q_{42},
\end{aligned}
$$

with $q_{i j}$ is the flow of the tank $i$ in the tank $j, q_{i j}=s_{i j} \sqrt{2 g h_{i}}$, $i \in\{3,4\}, j \in\{1,2\}, s_{i j}$ is section leak between tank $i$ and $j$, and $Q_{k}$ is flow of the pump $k, k \in\{1,2\}$. Then we replace $q_{i j}$ in (42) which gives

$$
\begin{aligned}
& \frac{d h_{1}}{d t}=-\frac{s_{3}}{S} \sqrt{2 g h_{1}}+\frac{s_{31}}{S} \sqrt{2 g h_{3}}+\frac{s_{41}}{S} \sqrt{2 g h_{4}}, \\
& \frac{d h_{2}}{d t}=-\frac{s_{4}}{S} \sqrt{2 g h_{2}}+\frac{s_{32}}{S} \sqrt{2 g h_{3}}+\frac{s_{42}}{S} \sqrt{2 g h_{4}},
\end{aligned}
$$




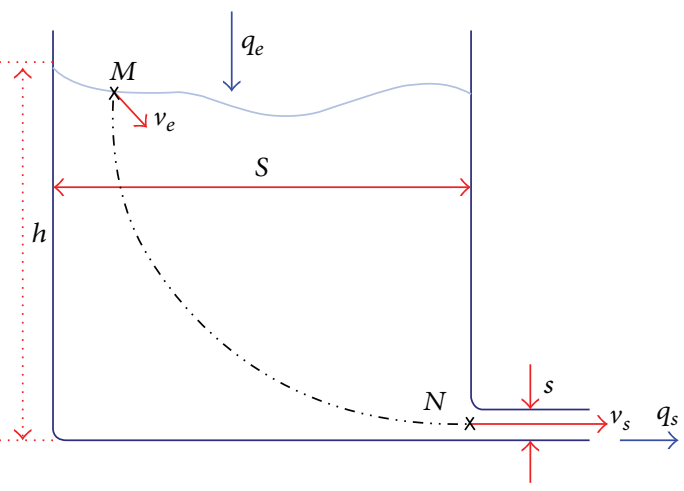

FIgURE 3: Elementary tank.

$$
\begin{aligned}
& \frac{d h_{3}}{d t}=-\frac{\left(s_{31}+s_{32}\right)}{S} \sqrt{2 g h_{3}}+\frac{1}{S} Q_{1}, \\
& \frac{d h_{4}}{d t}=-\frac{\left(s_{41}+s_{42}\right)}{S} \sqrt{2 g h_{4}}+\frac{1}{S} Q_{2} .
\end{aligned}
$$

To simplify the notations, let us rewrite (43) as follows:

$$
\begin{aligned}
& \dot{h}_{1}=-c_{1} \sqrt{h_{1}}+c_{2} \sqrt{h_{3}}+c_{3} \sqrt{h_{4}}, \\
& \dot{h}_{2}=-c_{4} \sqrt{h_{2}}+c_{5} \sqrt{h_{3}}+c_{6} \sqrt{h_{4}}, \\
& \dot{h}_{3}=-c_{7} \sqrt{h_{3}}+c_{8} U_{1}, \\
& \dot{h}_{4}=-c_{9} \sqrt{h_{4}}+c_{10} U_{2},
\end{aligned}
$$

where $c_{i}, i \in\{1, \ldots 10\}$ are constants summarized in Table 1 .

(B) Linearization. In the linearization phase, we fix an operating point $Q_{10}, Q_{20}, h_{0}=\left[\begin{array}{llll}h_{10} & h_{20} & h_{30} & h_{40}\end{array}\right]$.

We set $h_{i}=H_{i}+h_{i 0}(i=1, \ldots, 4)$ and $Q_{j}=U_{j}+q_{j 0} \quad(j=$ $1,2)$, where $H_{i}$ and $U_{j}$ are the variations around the operating point.

The system (44) becomes

$$
\begin{aligned}
\frac{d\left(H_{1}+h_{10}\right)}{d t}= & -\frac{s_{3}}{S} \sqrt{2 g\left(H_{1}+h_{10}\right)}+\frac{s_{31}}{S} \sqrt{2 g\left(H_{3}+h_{30}\right)} \\
& +\frac{s_{41}}{S} \sqrt{2 g\left(H_{4}+h_{40}\right)}, \\
\frac{d\left(H_{2}+h_{20}\right)}{d t}= & -\frac{s_{4}}{S} \sqrt{2 g\left(H_{2}+h_{20}\right)}+\frac{s_{32}}{S} \sqrt{2 g\left(H_{3}+h_{30}\right)} \\
& +\frac{s_{42}}{S} \sqrt{2 g\left(H_{4}+h_{40}\right)}, \\
\frac{d\left(H_{3}+h_{30}\right)}{d t}=- & \frac{\left(s_{31}+s_{32}\right)}{S} \sqrt{2 g\left(H_{3}+h_{30}\right)}+\frac{1}{S}\left(U_{1}+Q_{10}\right), \\
\frac{d\left(H_{4}+h_{40}\right)}{d t}=- & \frac{\left(s_{41}+s_{42}\right)}{S} \sqrt{2 g\left(H+h_{40}\right)}+\frac{1}{S}\left(U_{2}+Q_{20}\right) .
\end{aligned}
$$

Using

$$
\sqrt{1+x}=1+\frac{x}{2} \quad \text { when } x \ll 1
$$

TABLE 1: Four tanks system constants.

\begin{tabular}{lc}
\hline Constants & Numerical values \\
\hline$c_{1}, c_{4}$ & 0.0119 \\
$c_{2}, c_{6}$ & 0.014951 \\
$c_{3}, c_{5}$ & 0.002 \\
$c_{7}, c_{9}$ & 0.020333 \\
$c_{8}, c_{10}$ & 4.3 \\
\hline
\end{tabular}

and since $h_{i 0}=c s t e$, then $d h_{i 0} / d t=0$; we have

$$
\begin{aligned}
& \frac{d H_{1}}{d t}=-\frac{s_{3}}{S} \sqrt{\frac{g}{2 h_{10}}} H_{1}+\frac{s_{31}}{S} \sqrt{\frac{g}{2 h_{30}}} H_{3}+\frac{s_{41}}{S} \sqrt{\frac{g}{2 h_{40}}} H_{4}, \\
& \frac{d H_{2}}{d t}=-\frac{s_{4}}{S} \sqrt{\frac{g}{2 h_{20}}} H_{2}+\frac{s_{32}}{S} \sqrt{\frac{g}{2 h_{30}} H_{3}}+\frac{s_{42}}{S} \sqrt{\frac{g}{2 h_{40}}} H_{4}, \\
& \frac{d H_{3}}{d t}=-\frac{\left(s_{31}+s_{32}\right)}{S} \sqrt{\frac{g}{2 h_{30}}} H_{3}+\frac{1}{S} U_{1}, \\
& \frac{d H_{4}}{d t}=-\frac{\left(s_{41}+s_{42}\right)}{S} \sqrt{\frac{g}{2 h_{40}}} H_{4}+\frac{1}{S} U_{2} .
\end{aligned}
$$

(C) Simulation's Results. The aim of this application is to maintain the water level in each tank below a threshold selected by the user during a given time interval. It will substantially stabilize the water level in the four tanks.

We linearize the system around the point

$$
h_{0}=\left[\begin{array}{llll}
0.3145 & 0.3918 & 0.15 & 0.2
\end{array}\right]^{T} \text {. }
$$

We obtain the linear system

$$
\begin{aligned}
\dot{h}(t)= & {\left[\begin{array}{cccc}
-0.0106 & 0 & 0.0193 & 0.0022 \\
0 & -0.0095 & 0.0026 & 0.0167 \\
0 & 0 & -0.0262 & 0 \\
0 & 0 & 0 & -0.0227
\end{array}\right] h(t) } \\
& +\left[\begin{array}{cc}
0 & 0 \\
0 & 0 \\
4.3 & 0 \\
0 & 4.3
\end{array}\right] u(t),
\end{aligned}
$$

where $h(t)$ is the state vector.

The constants $c_{1}, c_{2}$, and $T$ are chosen as $c_{1}=0.7, c_{2}=0.8$, and $T=200 s$.

The controller $K$ is provided by Theorem 4 as

$$
K=\left(\begin{array}{cccc}
-0.0047 & -0.0006 & 0.0205 & 0 \\
-0.0005 & -0.0041 & 0 & -0.0352
\end{array}\right)
$$

with

$$
R=\left(\begin{array}{cccc}
-0.0150 & -0.0020 & 0.0628 & 0 \\
-0.0016 & -0.0120 & 0 & -0.1079
\end{array}\right)
$$

$$
S=\left(\begin{array}{cccc}
3.0880 & 0 & -0.0193 & -0.0022 \\
0 & 3.0859 & -0.0026 & -0.0168 \\
-0.0193 & -0.0026 & 3.0668 & 0 \\
-0.0022 & -0.0168 & 0 & 3.0668
\end{array}\right)
$$




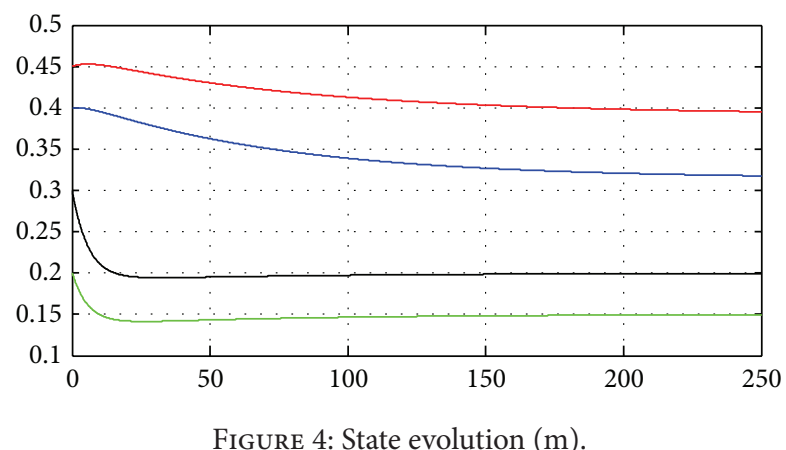

Figure 4 shows the evolution of the state, Figure 5 shows the evolution of the norm of the state, and Figure 6 shows the evolution of both control components which represent the flows $Q_{1}$ (blue) and $Q_{2}$ (red) of both water pumps.

\section{Case of Uncertain System}

A certain knowledge of the state model is necessary for the stabilization in finite time by state feedback, but this is not always the case. In fact, the model parameters may change over time. Models are usually obtained from an identification that uses measurements (input-output) where the measurement noise and errors cause uncertainties in the model. In this context, a robust command can provide the satisfaction of a number of performances although there exist uncertainties in the model. We propose in this section a theorem in which finite time stabilizes uncertain system and takes into account parameters uncertainties. The polytopic uncertainties as well as the norm bounded ones are considered.

5.1. Polytopic Uncertainty. We consider in this paragraph a system described by

$$
\dot{x}=A(\alpha) x(t)+B(\theta) u(t),
$$

where $x \in \mathbb{R}^{n}$ is the state vector. The dynamic matrix $A(\alpha)$ and the input matrix $B(\theta)$ belong respectively to the following sets:

$$
\begin{aligned}
& \mathscr{A}=\left\{A(\alpha): A(\alpha)=\sum_{i=1}^{N_{A}} \alpha_{i} A_{i}, \sum_{i=1}^{N_{A}} \alpha_{i}=1, \alpha_{i} \geq 0\right\}, \\
& \mathscr{B}=\left\{B(\theta): B(\theta)=\sum_{j=1}^{N_{B}} \theta_{j} B_{j}, \sum_{j=1}^{N_{B}} \theta_{j}=1, \theta_{j} \geq 0\right\} .
\end{aligned}
$$

In the following, we extend the previous results given for the certain systems to the case of uncertain ones. This can be achieved by tuning Theorem 8 as follows.

Theorem 10. If there exist a positive definite symmetric matrix $S$, a matrix $R$ of appropriate dimensions, and a positive scalar $\beta$ such that

$$
0 \leq \beta T \leq \ln \left[\frac{c_{2}}{c_{1}}\right]
$$

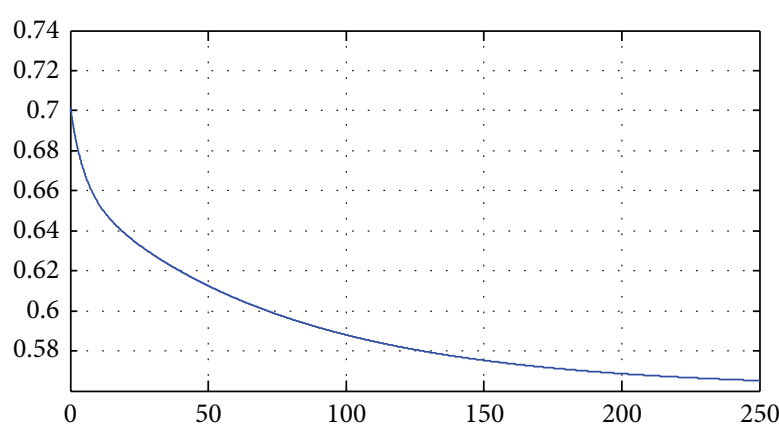

FIGURE 5: Norm state evolution.

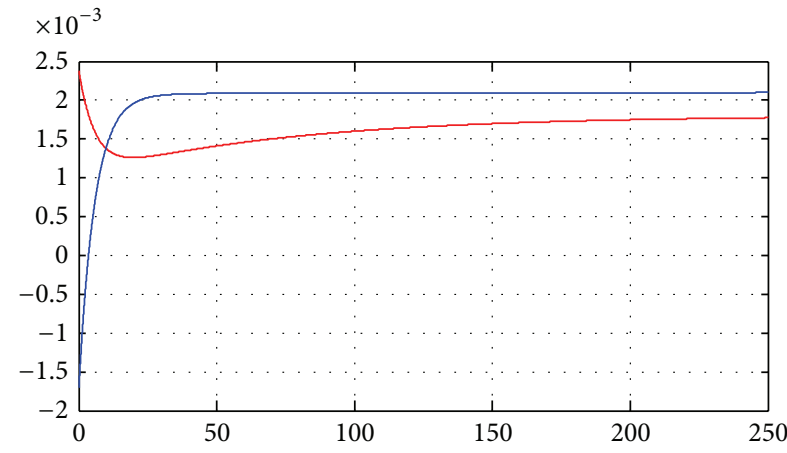

Figure 6: Control evolution (L/min).

and satisfying the matrix inequalities

$$
\begin{gathered}
A_{i} S+S A_{i}^{T}+B_{j} R+R^{T} B_{j}^{T}-\beta I \leq 0, \\
i=1, \ldots, N_{A}, \quad j=1, \ldots, N_{B}, \\
I \leq S \leq \frac{c_{2}^{2}}{c_{1}^{2}} e^{-\beta T} I,
\end{gathered}
$$

then the control law $u=R S^{-1} \times$ finite time stabilizes the system (52)-((53a) and (53b)).

Proof. The proof is simple as long as we can apply Corollary 9 to the system (52)-((53a) and (53b)) by replacing $(A+B K)$ by $\left(A_{i}+B_{j} K\right)$, taking into account the properties of convexity.

5.2. Norm Bounded Uncertainty. Another uncertainty description is as follows:

$$
\dot{x}(t)=\left(A+D F E_{1}\right) x(t)+\left(B+D F E_{2}\right) u(t),
$$

with

$$
F^{T} F \leq I
$$

We have the following result.

Theorem 11. If there exist a positive definite symmetric matrix $S$, a matrix $R$ of appropriate dimensions, and a positive scalars $\beta$, $\varepsilon$ such that

$$
0 \leq \beta T \leq 2 \ln \left[\frac{c_{2}}{c_{1}}\right]
$$


and satisfying the matrix inequalities

$$
\begin{gathered}
\left(\begin{array}{cc}
A S+S A^{T}+B R+R^{T} B^{T}+\varepsilon D D^{T}-\beta I & \left(E_{1} S+E_{2} R\right)^{T} \\
E_{1} S+E_{2} R & -\varepsilon I
\end{array}\right) \leq 0 \\
I \leq S \leq \frac{c_{2}^{2}}{c_{1}^{2}} e^{-\beta T} I
\end{gathered}
$$

then the control law $u=R S^{-1} \times$ finite time stabilizes the system (56).

Proof. By Schur complement, (59) is equivalent to

$$
\begin{aligned}
A S & +S A^{T}+B R+R^{T} B^{T}+\varepsilon D D^{T} \\
& +\varepsilon^{-1}\left(E_{1} S+E_{2} R\right)^{T}\left(E_{1} S+E_{2} R\right)-\beta I \leq 0
\end{aligned}
$$

which can be written as

$$
\begin{aligned}
(A & \left.+B R S^{-1}\right) S+S\left(A+B R S^{-1}\right)^{T}+\varepsilon D D^{T} \\
& +\varepsilon^{-1} S\left(E_{1}+E_{2} R S^{-1}\right)^{T}\left(E_{1}+E_{2} R S^{-1}\right) S-\beta I \leq 0 .
\end{aligned}
$$

Taking into account the uncertainties of (56),

$$
\begin{aligned}
(A & \left.+B R S^{-1}+D F\left(E_{1}+E_{2} R S^{-1}\right)\right) S \\
& +S\left(A+B R S^{-1}+D F\left(E_{1}+E_{2} R S^{-1}\right)\right)^{T}-\beta I \\
= & \left(A+B K+D F\left(E_{1}+E_{2} K\right)\right) S \\
& +S\left(A+B K+D F\left(E_{1}+E_{2} K\right)\right)^{T}-\beta I \\
\leq & \left(A+B R S^{-1}\right) S+S\left(A+B R S^{-1}\right)^{T}+\varepsilon D D^{T} \\
& +\varepsilon^{-1} S\left(E_{1}+E_{2} R S^{-1}\right)^{T}\left(E_{1}+E_{2} R S^{-1}\right) S-\beta I \leq 0
\end{aligned}
$$

which concludes the proof.

Remark 12. In [11] the authors solved the finite times stabilization problem for the uncertain systems by using the Control Lyapunov Functions (CLF), but in this paper we solve the problem by using Linear Matrix Inequalities (LMIs).

\section{Conclusion}

The problem of finite time stabilization of linear certain and uncertain systems is solved by giving an explicit formula for a state feedback control law. This latter one is based on solving some Linear Matrix Inequalities. The proposed approach satisfies certain simple conditions and the control law provides, for certain and uncertain linear systems, the constraint on the norm of the state during the fixed time interval. An application to the four tanks system shows the efficiency of the proposed approach. Since the state of the system is not always measurable, we can treat the case of the finite time controller of linear and nonlinear systems by output feedback. The results proposed in this paper can be applied to vehicle suspension control systems to improve their performances.

\section{Conflict of Interests}

The authors declare that there is no conflict of interests regarding the publication of this paper.

\section{References}

[1] S. Tarbouriech and G. Garcia, Control of Uncertain Systems with Bounded Inputs, Springer, Berlin, Germany, 1997.

[2] S. P. Bhat and D. S. Bernstein, "Continuous finite-time stabilization of the translational and rotational double integrators," IEEE Transactions on Automatic Control, vol. 43, no. 5, pp. 678-682, 1998.

[3] P. M. Abhilash and A. D. Mahindrakar, "Stabilization of a circular ball-and-beam system with input and state constraints using linear matrix inequalities," in Proceedings of the IEEE International Conference on Systems, Man and Cybernetics (SMC '08), pp. 3201-3205, Singapore, October 2008.

[4] P. Dorato, "Short time stability in linear time-varying systems," in Proceedings of the IRE International Convention Record Part 4, pp. 83-87, New York, NY, USA, 1961.

[5] S. Boyd, L. El Ghaoui, E. Feron, and V. Balakrishnan, Linear Matrix Inequalities in System and Control Theory, SIAM Press, Philadelphia, Pa, USA, 1994.

[6] S. P. Bhattacharyya, H. Chapellat, and L. H. Keel, Robust Control: The Parametric Approach, Prentice Hall, Upper Saddle River, NJ, USA, 1995.

[7] T. H. Gronwall, "Note on the derivatives with respect to a parameter of the solutions of a system of differential equations," Annals of Mathematics, vol. 20, no. 4, pp. 292-296, 1919.

[8] L. Weiss and E. F. Infante, "Finite time stability under perturbing forces and on product spaces," IEEE Transactions on Automatic Control, vol. 12, no. 1, pp. 54-59, 1967.

[9] E. Moulay, Contribution à l'étude de la stabilité en temps fini et de la stabilisation [Thèse de Doctorat], Ecole centrale de Lille, 2005.

[10] W. B. Mabrouk, C. B. Njima, H. Messaoud, and G. Garcia, "Finite-time stabilization of nonlinear affine systems," Journal Européen des Systèmes Automatisés, vol. 44, no. 3, pp. 327-343, 2010.

[11] C. B. Njima, W. B. Mabrouk, G. Garcia, and H. Messaoud, "Robust finite-time stabilization of nonlinear systems," International Review of Automatic Control, vol. 4, no. 3, pp. 362-369, 2011.

[12] G. Garcia, S. Tarbouriech, and J. Bernussou, "Finite-time stabilization of linear time-varying continuous systems," IEEE Transactions on Automatic Control, vol. 54, no. 2, pp. 364-369, 2009.

[13] L. T. Grujic, "On practical stability," International Journal of Control, vol. 17, no. 4, pp. 881-887, 1973. 


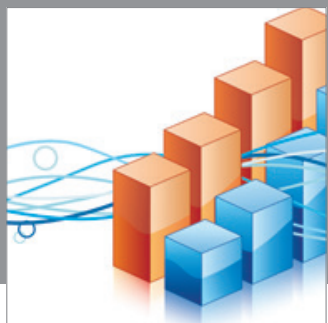

Advances in

Operations Research

mansans

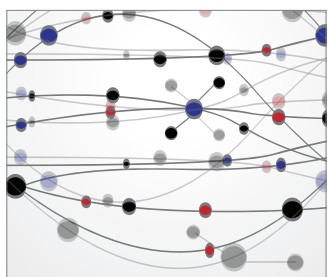

The Scientific World Journal
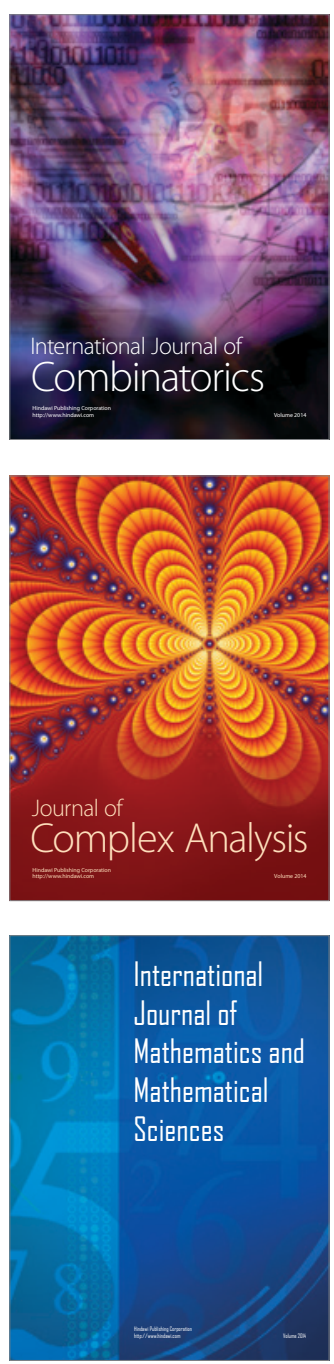
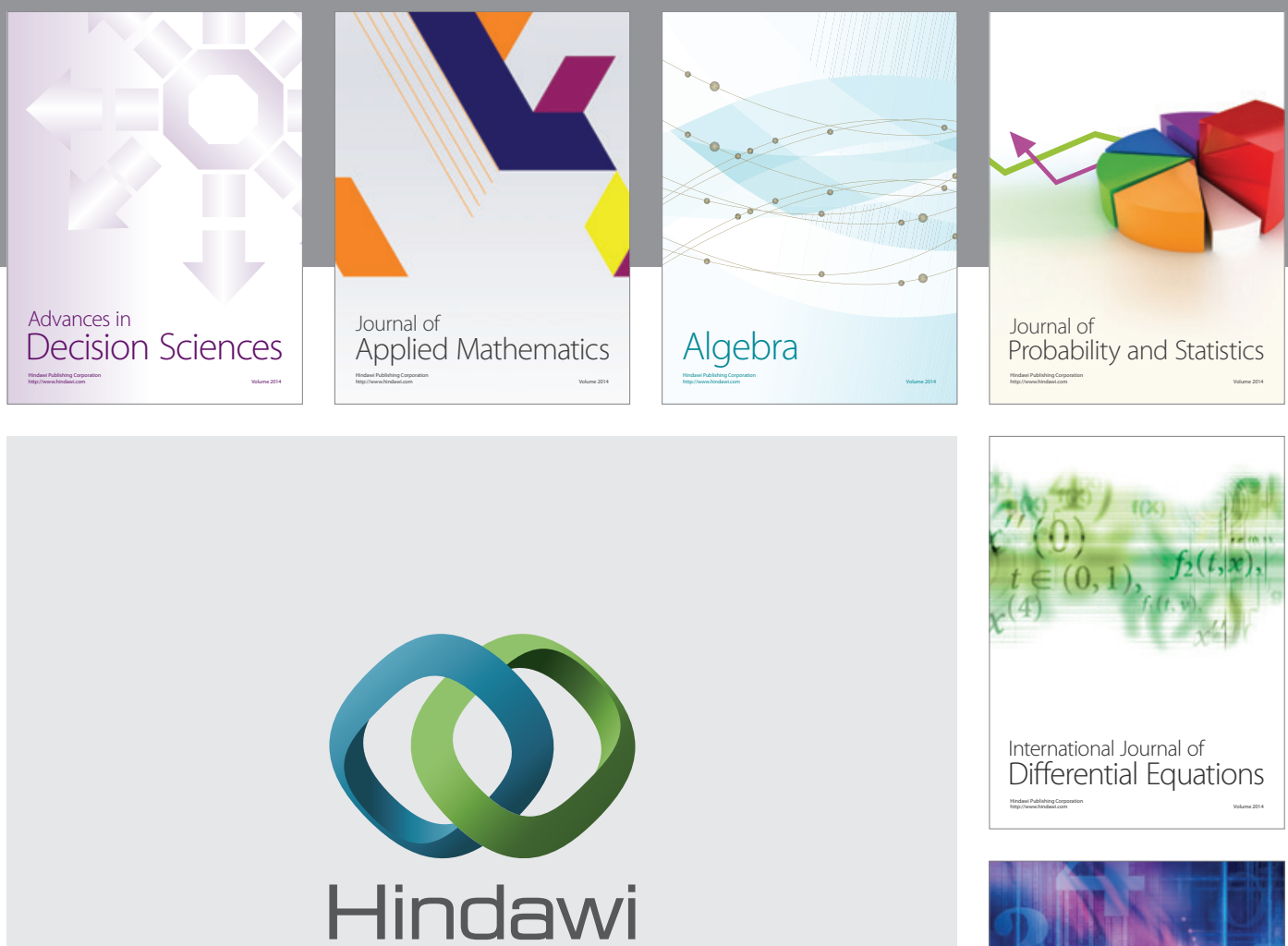

Submit your manuscripts at http://www.hindawi.com
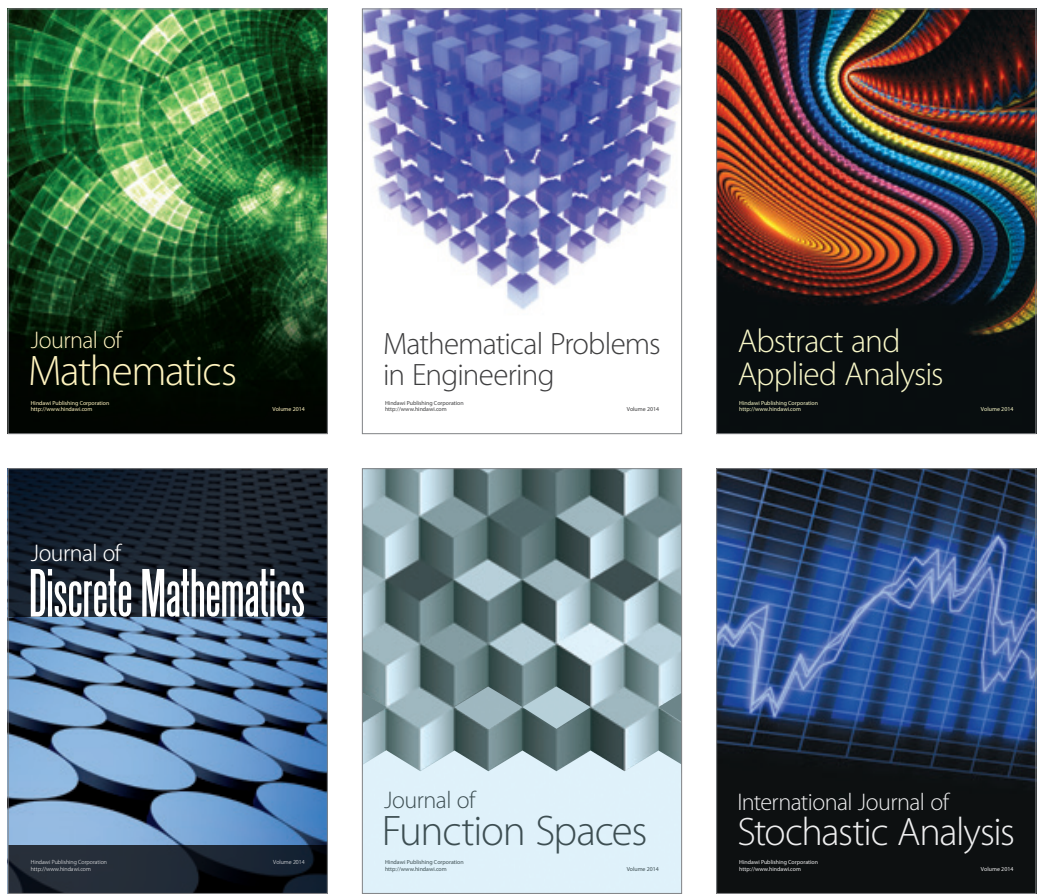

Journal of

Function Spaces

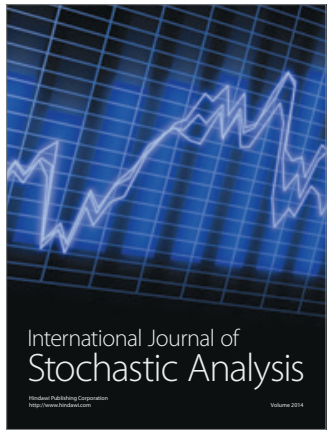

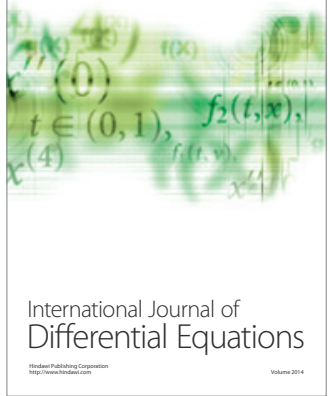
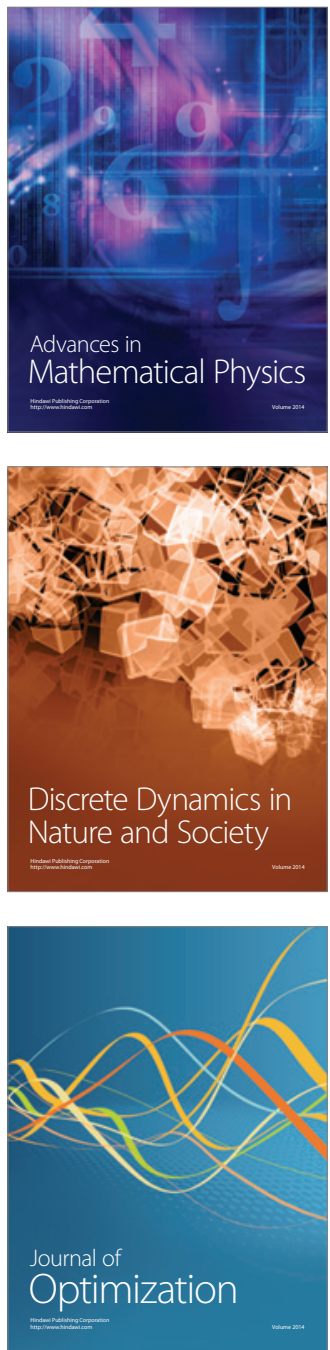